\title{
Las categorías o facetas fundamentales: una metodología para el diseño de taxonomías corporativas de sitios $W e b$ argentinos
}

Ana Martínez

Profesora titular de organización del conocimiento I. Departamento de Bibliotecología. Facultad de Humanidades y Ciencias de la Educación, Universidad Nacional de La Plata

E-mail: ammarti@speedy.com.ar

\section{Cristina Ristuccia}

Profesora titular de catalogación I, Departamento de Bibliotecología. Facultad de Humanidades y Ciencias de la Educación, Universidad Nacional de La Plata

E-mail: dhubi@fahce.unlp.edu.ar

\section{Rosa Pisarello}

Profesora titular de referencia general, Departamento de Bibliotecología. Facultad de Humanidades y Ciencias de la Educación, Universidad Nacional de La Plata

\section{Edgardo Stubbs}

Ayudante diplomado de catalogación I, Departamento de Bibliotecología. Facultad de Humanidades y Ciencias de la Educación, Universidad Nacional de La Plata

\section{Laura Caminotti}

Adscripta a la cátedra de documentación general, Departamento de Bibliotecología. Facultad de Humanidades y Ciencias de la Educación, Universidad Nacional de La Plata

\section{José Balparda}

Adscripto a la cátedra de organización del conocimiento I, Departamento de Bibliotecología. Facultad de Humanidades y Ciencias de la Educación, Universidad Nacional de La Plata

Julia Valdez

Ayudante diplomado de organización del conocimiento I, Departamento de Bibliotecología. Facultad de Humanidades y Ciencias de la Educación, Universidad Nacional de La Plata

\section{Norma Mangiaterra}

Profesora titular de documentación general, Departamento de Bibliotecología. Facultad de Humanidades y Ciencias de la Educación, Universidad Nacional de La Plata

\section{Resumen}

El análisis por facetas ha sido revaloado en los últimos años para el diseño de taxonomías corporativas. Con el fin de confirmar su utilidad, analizamos los sitios Web de diferentes organizaciones argentinas: tres bodegas de vino, tres industrias alimenticias, seis clubes de fútbol, cinco asociaciones profesionales, tres facultades universitarias y cinco agencias de gobierno. De las 16 categorías o facetas fundamentales propuestas por el Classification Research Group, las bodegas de vino coincidieron en 13 (81\%), las industrias alimenticias en 11 (68\%), los clubes de fútbol en 7 (43\%), las asociaciones profesionales en 5 (31\%), las facultades en 8 (50\%) y las agencias de gobierno en 9 (56\%). Estas diferencias se deben al hecho de que no todas las categorías son necesarias para todas las organizaciones. Se concluye que el método del análisis por facetas es útil para diseñar taxonomías corporativas y optimizar los sitios Web, de acuerdo con las características particulares de cada organización.

\section{Palabras claves}

Análisis de facetas; Taxonomías corporativas; Sítios Web argentinos.

\section{Fundamental categories or facets: a methodology for the design of corporate taxonomies of Argentinian web sites}

\begin{abstract}
Faceted analysis has been reassessed in last years for designing corporative taxonomies. In order to confirm its usefulness, we analyzed the Web sites of different Argentine organizations: three wineries, three food industries, six football clubs, five professional associations, three university schools, and five government agencies. From the 16 fundamental categories or facets proposed by the Classification Research Group, wineries coincide in 13 (81\%), food industries in 11 (68\%), football clubs in 7 (43\%), professional associations in 5 (31\%), university schools in 8 (50\%), and government agencies in 9 (56\%). These differences are due to the fact that not all the categories are necessary for all organizations. We conclude that the method of faceted analysis is useful for designing corporative taxonomies and for optimizing Web sites, in accordance to particular characteristics of each organization.
\end{abstract}

\section{Keywords}

Faceted analysis; Corporative taxonomies; Argentine Web sites. 
Las categorías o facetas fundamentales: una metodología para el diseño de taxonomías corporativas...

\section{INTRODUCCIÓN}

En los últimos años, diversos sistemas de organización del conocimiento (SOC) se han utilizado con éxito y recomendado para ordenar la información en la World Wide Web: desde los tradicionales sistemas de clasificación y tesauros, hasta las más novedosas taxonomías, ontologías y redes semánticas (1-6). De este modo, el término taxonomía (del griego taxis $=$ orden y onoma $=$ nombre) que es un sinónimo de clasificación (7) de uso común en las ciencias naturales, se ha ido imponiendo en el diseño de sitios Web.

Para Gilchrist (8) la taxonomía es, en un sentido amplio, la creación de la estructura (orden) y de los rótulos (nombres) que ayudan a localizar la información relevante. En un sentido más específico, es el ordenamiento y rotulación de los metadatos, que permiten gestionar sistemáticamente la información primaria.

Por otra parte, algunos autores han propuesto el método del análisis por facetas concebido por el bibliotecario indio Shiyali Ramamrita Ranganathan (9) para el diseño de taxonomías Web. Entre los trabajos sobre este particular se pueden mencionar los de Tudhope et al. (10), Broughton (11), Louis (12), Priss (13-15) y Priss y Jacob (16).

El diseño de un SOC facetado se inicia analizando el universo del conocimiento mediante un conjunto de categorías o facetas fundamentales. Barité (17) define las categorías fundamentales como "abstracciones simplificadas que, con fuerza de herramientas intelectuales, son usadas por los clasificacionistas para indagar las regularidades de los objetos del mundo físico y del ideal y de las nociones que los representan, con el objeto de organizar lógicamente sistemas de conceptos aptos para la organización del conocimiento en general, y la clasificación documental en particular". Tienen utilidad en el diseño, planeamiento y estructuración de los SOC, así como para su modificación y evaluación (17).

Ranganathan (9) postuló cinco categorías fundamentales: Personalidad (P), Materia (M), Energía (E), Espacio (S) y Tiempo ( $T$ ), en un orden de concretidad decreciente PMEST, donde $\mathrm{P}$ es la categoría más concreta y $\mathrm{T}$ la menos concreta. Según el mismo autor, estas categorías fundamentales son aplicables a todos los campos del conocimiento por igual.

Los seguidores británicos de Ranganathan, que fundaron en 1952 el Classification Research Group (CRG), han profundizado y optimizado el método del análisis por facetas, aplicándolo al diseño de sistemas de clasificación, tesauros y epígrafes facetados e incluso a la utilización de la lengua natural con fines de recuperación de información como el sistema PRECIS (18). A diferencia de Ranganathan, el CRG no considera que un único conjunto de categorías fundamentales pueda aplicarse por igual a todos los campos temáticos, sino que para cada campo pueden y deben identificarse primero las categorías fundamentales más adecuadas. Sin embargo, a lo largo de sus trabajos, los miembros del CRG han utilizado con frecuencia varias de estas categorías, aplicadas a distintos temas (19).

En nuestro país no existen experiencias publicadas sobre el diseño de SOC facetados y menos aún de su aplicación a las taxonomías Web. Por este motivo, el propósito del presente trabajo ha sido la identificación de las categorías fundamentales que subyacen a diversas taxonomías de sitios Web correspondientes a organizaciones argentinas de distinto tipo, con el fin de determinar si coinciden o no con las categorías fundamentales del CRG y, en tal caso, si éstas pueden recomendarse para el diseño de sitios Web organizacionales.

\section{METODOLOGÍA}

Se estudiaron 25 sitios Web de organizaciones argentinas de diversos rubros, cuyas taxonomías no han sido diseñadas con el método del análisis por facetas de modo premeditado.

Se analizó la taxonomía de cada uno de los sitios Web, identificándose las clases o taxones de primero y segundo nivel, es decir las clases más generales. Cada una de las clases identificadas fue clasificada bajo una de las categorías o facetas fundamentales usadas por el CRG $(17,19$, que se muestran en la tabla 1 , en seguida.

A continuación se agruparon todas las clases clasificadas bajo sus respectivas categorías fundamentales, según los distintos rubros. Se obtuvieron así seis grupso de sitios Web:

a. Bodegas de vino: tres sitios Web.

b. Industrias alimenticias: tres sitios Web.

c. Clubes de fútbol: seis sitios Web.

d. Asociaciones profesionales: cinco sitios Web.

e. Facultades universitarias: tres sitios Web.

f. Agencias de gobierno: cinco sitios web.

Finalmente, se compararon los seis grupos para determinar en qué medida las categorías fundamentales del CRG resultaban comunes a los distintos rubros. 


\section{Ana Martínez / Cristina Ristuccia / Rosa Pisarello / Edgardo Stubbs / Laura Caminotti / José Balparda / Julia Valdez / Norma Mangiaterra}

Algunas clases representaban en realidad más de un concepto y se clasificaron dos veces, por ejemplo: bajo la categoría Productos finales se incluyeron Productos para Argentina y Productos de exportación, pero además se clasificó Argentina bajo la categoría Espacio y Exportación bajo Operaciones. De igual manera Servicios a los afiliados y Servicios a la comunidad se clasificaron en Operaciones, pero los conceptos Afiliados y Comunidad se clasificaron también bajo Pacientes.

En el caso de los clubes de fútbol, bajo los nombres de las distintas competencias se incluían tanto la participación presente en dichas competencias, que se incluyó en Operaciones, como los títulos obtenidos que se clasificaron en Productos finales.

\section{RESULTADOS Y DISCUSIÓN}

En la tabla 2, en seguida, se muestran las clases o taxones clasificados bajo las categorías correspondientes a Entidades/cosas/objetos por características. De las ocho categorías fundamentales del CRG analizadas en esta tabla, las bodegas de vino incluyen $5(62,5 \%)$, las industrias alimenticias 4 (50\%), los clubes de fútbol 2 (25\%), las asociaciones profesionales 0 , las facultades 1 $(12,5 \%)$ y las agencias de gobierno 2 (25\%).

En la tabla 3, en seguida, se muestran las clases o taxones clasificados bajo las categorías correspondientes a Entidades/cosas/objetos por función. De las cuatro categorías fundamentales del CRG analizadas en esta tabla, todos los grupos incluyen 3 (75\%).

Las clases o taxones clasificados por Acciones/actividades se presentan en la tabla 4, en seguida. De las dos categorías fundamentales del CRG analizadas en esta tabla, las bodegas de vino, industrias alimenticias, facultades y agencias de gobierno incluyen ambas (100\%), mientras que los clubes de fútbol y las asociaciones profesionales sólo incluyen 1 .

Las clases o taxones correspondientes a Espacio y Tiempo se pueden observar en la tabla 5 , en seguida. De las dos categorías fundamentales del CRG analizadas en esta tabla, las bodegas de vino, industrias alimenticias, facultades y agencias de gobierno incluyen ambas (100\%), mientras que los clubes de fútbol y las asociaciones profesionales sólo incluyen 1 .
TABLA 1

Categorías fundamentales del CRG utilizadas para el análisis de sitios Web argentinos (tomado de Aitchison et alii., 19)

\begin{tabular}{|c|c|}
\hline Categorías fundamentales & Ejemplos \\
\hline Entidades, cosas u objetos & \\
\hline [por características] & \\
\hline Entidades abstractas & Ideas, disciplinas \\
\hline Entidades de la naturaleza & Radiación, nubes, ríos \\
\hline Entidades u organismos vivos & Bacterias, virus, mamíferos \\
\hline $\begin{array}{l}\text { Artefactos hechos por el } \\
\text { hombre }\end{array}$ & Manufacturas, objetos de arte \\
\hline $\begin{array}{l}\text { Atributos: propiedades, } \\
\text { cualidades, estados, } \\
\text { condiciones }\end{array}$ & $\begin{array}{l}\text { Temperatura, color, } \\
\text { confiabilidad, tamaño }\end{array}$ \\
\hline $\begin{array}{l}\text { Materiales o sustancias } \\
\text { constituyentes }\end{array}$ & $\begin{array}{l}\text { Minerales, madera, cuero, } \\
\text { plásticos, drogas }\end{array}$ \\
\hline Partes o componentes & $\begin{array}{l}\text { Timones, partes del cuerpo, } \\
\text { techos, pisos }\end{array}$ \\
\hline $\begin{array}{l}\text { Entidades completas o } \\
\text { complejas }\end{array}$ & $\begin{array}{l}\text { Vehículos, barcos, edificios, } \\
\text { escuelas }\end{array}$ \\
\hline \multicolumn{2}{|l|}{ [por función] } \\
\hline \multicolumn{2}{|l|}{$\begin{array}{l}\text { Agentes (realizadores de una } \\
\text { acción, animados o } \\
\text { inanimados): }\end{array}$} \\
\hline $\begin{array}{l}\text { Individuos, personal, } \\
\text { organizaciones }\end{array}$ & $\begin{array}{l}\text { Artistas, marinos, doctores, } \\
\text { asociaciones profesionales }\end{array}$ \\
\hline Equipos, aparatos & $\begin{array}{l}\text { Máquinas, computadoras, } \\
\text { respiradores, lavavajillas }\end{array}$ \\
\hline $\begin{array}{l}\text { Pacientes (receptores de una } \\
\text { acción, animados o } \\
\text { inanimados) }\end{array}$ & $\begin{array}{l}\text { Víctimas, beneficiarios, } \\
\text { audiencias }\end{array}$ \\
\hline Productos finales & $\begin{array}{l}\text { Productos alimenticios, } \\
\text { residuos, resultados de una } \\
\text { investigación }\end{array}$ \\
\hline Acciones o actividades & $\begin{array}{l}\text { Desarrollo, enfermedades, } \\
\text { pensamiento, caminata }\end{array}$ \\
\hline $\begin{array}{l}\text { Procesos o funciones (procesos } \\
\text { internos, acciones } \\
\text { intransitivas) }\end{array}$ & \\
\hline $\begin{array}{l}\text { Operaciones (externas, } \\
\text { acciones transitivas) }\end{array}$ & $\begin{array}{l}\text { Enseñanza, calentamiento, } \\
\text { gestión }\end{array}$ \\
\hline \multicolumn{2}{|l|}{$\begin{array}{l}\text { Espacio, lugar, locación, } \\
\text { ambiente }\end{array}$} \\
\hline Tiempo & \\
\hline
\end{tabular}


Las categorías o facetas fundamentales: una metodología para el diseño de taxonomías corporativas...

TABLA 2

Entidades, cosas u objetos por características

\begin{tabular}{|c|c|c|c|c|c|c|}
\hline Categoria & Vinos & Alimentos & Fútbol & Asociaciones & Facultades & Gobierno \\
\hline Entidades abstractas & - & $\begin{array}{c}\text { Filosofía corporativa } \\
\text { Objetivos } \\
\text { Visión } \\
\text { Misión } \\
\text { Liderazgo } \\
\text { Política de calidad } \\
\end{array}$ & - & - & - & Políticas \\
\hline $\begin{array}{l}\text { Entidades de la } \\
\text { naturaleza }\end{array}$ & Uvas & $\begin{array}{c}\text { Leche } \\
\text { Ingredientes } \\
\end{array}$ & - & - & - & - \\
\hline $\begin{array}{l}\text { Entidades u } \\
\text { organismos vivos }\end{array}$ & - & - & - & - & - & - \\
\hline $\begin{array}{l}\text { Artefactos hechos por } \\
\text { el hombre }\end{array}$ & $\begin{array}{l}\text { Barricas } \\
\text { Barriles } \\
\text { Vasijas }\end{array}$ & - & $\begin{array}{c}\text { Camisetas } \\
\text { Emblemas } \\
\text { Fotos } \\
\text { Tarjetas } \\
\text { Graffitis } \\
\end{array}$ & - & - & - \\
\hline $\begin{array}{l}\text { Atributos, propiedades, } \\
\text { cualidades, estados y } \\
\text { condiciones }\end{array}$ & $\begin{array}{c}\text { Color } \\
\text { Sabor } \\
\text { Gusto } \\
\text { Aroma } \\
\text { Temperatura } \\
\text { Añejamiento } \\
\text { Presentación } \\
\end{array}$ & $\begin{array}{c}\text { Tipos } \\
\text { Sabores } \\
\text { Valor nutricional } \\
\text { Presentación }\end{array}$ & - & - & - & - \\
\hline $\begin{array}{l}\text { Materiales o sustancias } \\
\text { constituyentes }\end{array}$ & $\begin{array}{l}\text { Alcohol } \\
\text { Azúcar }\end{array}$ & - & - & - & - & - \\
\hline \multicolumn{7}{|l|}{ Partes o componentes } \\
\hline $\begin{array}{l}\text { Entidades completas } \\
\text { (todo) o complejas }\end{array}$ & $\begin{array}{l}\text { Bodegas } \\
\text { Viñedos } \\
\text { Fincas }\end{array}$ & Plantas industriales & $\begin{array}{c}\text { Estadio } \\
\text { Instalaciones } \\
\text { Sede social } \\
\text { Museo }\end{array}$ & - & $\begin{array}{c}\text { Biblioteca } \\
\text { Centro multimedia } \\
\text { Hospital escuela }\end{array}$ & $\begin{array}{c}\text { Centro de } \\
\text { documentación }\end{array}$ \\
\hline
\end{tabular}

TABLA 3

Entidades, cosas u objetos por función

\begin{tabular}{|c|c|c|c|c|c|c|}
\hline Categoría & Vinos & Alimentos & Fútbol & Asociaciones & Facultades & Gobierno \\
\hline $\begin{array}{l}\text { Agentes } \\
\text { (individuos, personal, } \\
\text { organizaciones) }\end{array}$ & $\begin{array}{l}\text { Institución(al) } \\
\text { Directorio } \\
\text { Distribuidores } \\
\text { Representantes } \\
\text { comerciales }\end{array}$ & \begin{tabular}{|l|} 
Institución(al) \\
Estructura comercial \\
Estructura industrial \\
Estructura organizativa \\
Directorio \\
Recursos humanos
\end{tabular} & \begin{tabular}{|l|} 
Institución(al) \\
El Club \\
Departamentos \\
Filiales \\
Autoridades \\
Directivos \\
Presidentes \\
Comisión directiva \\
Cuerpo técnico \\
Técnicos \\
Equipo profesional \\
Plantel \\
Jugadores \\
Goleadores \\
\end{tabular} & $\begin{array}{l}\text { Institución(al) } \\
\text { Comisiones } \\
\text { Subcomisiones } \\
\text { Autoridades } \\
\text { Directorio }\end{array}$ & $\begin{array}{l}\text { Institución(al) } \\
\text { Secretarías } \\
\text { Cátedras } \\
\text { Autoridades } \\
\text { Consejo académico }\end{array}$ & $\begin{array}{l}\text { Autoridades } \\
\text { Secretarías } \\
\text { Subsecretarías } \\
\text { Direcciones } \\
\text { Policía } \\
\text { Proveedores }\end{array}$ \\
\hline $\begin{array}{l}\text { Agentes } \\
\text { (equipos, aparatos) }\end{array}$ & - & - & - & - & - & - \\
\hline Pacientes & Clientes & \begin{tabular}{|l|} 
Clientes \\
Consumidor \\
Comunidad \\
\end{tabular} & $\begin{array}{l}\text { Socios } \\
\text { Hinchada }\end{array}$ & \begin{tabular}{|l|} 
Membresía \\
Asociados \\
Comunidad \\
\end{tabular} & \begin{tabular}{|l|} 
Alumnos \\
Estudiantes \\
Terceros \\
\end{tabular} & Usuarios \\
\hline Productos finales & $\begin{array}{l}\text { Productos } \\
\text { Productos para } \\
\text { Argentina } \\
\text { Productos para } \\
\text { exportación } \\
\text { Guía del catador } \\
\text { Noticias } \\
\text { Novedades }\end{array}$ & $\begin{array}{l}\text { Productos } \\
\text { Ferias internacionales } \\
\text { Noticias } \\
\text { Novedades } \\
\text { Prensa }\end{array}$ & $\begin{array}{l}\text { [títulos obtenidos en] } \\
\text { Campeonatos } \\
\text { nacionales } \\
\text { Torneo Apertura } \\
\text { Copa Libertadores } \\
\text { Copa Intercontinental } \\
\text { Supercopa } \\
\text { Sudamericana }\end{array}$ & \begin{tabular}{|l|} 
Publicaciones \\
Boletines \\
Libros, videos, CD \\
Cursos \\
Bolsa de trabajo \\
Convenios \\
Reglamentos \\
Estatuto social \\
Código de ética \\
Informes \\
\end{tabular} & $\begin{array}{l}\text { Carreras de pregrado } \\
\text { Carreras de posgrado } \\
\text { Bases de datos } \\
\text { Información para } \\
\text { estudiantes }\end{array}$ & $\begin{array}{l}\text { Bases de datos } \\
\text { Publicaciones } \\
\text { Boletín } \\
\text { Boletín oficial } \\
\text { Sistema de expedientes } \\
\text { Resoluciones } \\
\text { Prensa } \\
\text { Leyes }\end{array}$ \\
\hline
\end{tabular}


Ana Martínez / Cristina Ristuccia / Rosa Pisarello / Edgardo Stubbs / Laura Caminotti / José Balparda / Julia Valdez / Norma Mangiaterra

TABLA 4

Acciones o actividades

\begin{tabular}{|c|c|c|c|c|c|c|}
\hline Categoría & Vinos & Alimentos & Fútbol & Asociaciones & Facultades & Gobierno \\
\hline Procesos o funciones & $\begin{array}{l}\text { Crianza del vino } \\
\text { Vinificación } \\
\text { Elaboración } \\
\text { Proceso de elaboración }\end{array}$ & \begin{tabular}{|l|} 
Calidad \\
Calidad hoy \\
Logística \\
Producción \\
Producción lechera \\
\end{tabular} & - & - & Concursos docentes & $\begin{array}{l}\text { Aspectos } \\
\text { presupuestarios } \\
\text { Presupuestaria }\end{array}$ \\
\hline Operaciones & $\begin{array}{l}\text { Mercado externo } \\
\text { Mercado interno } \\
\text { Exportación } \\
\text { Campañas } \\
\text { Visitas guiadas } \\
\text { Turismo }\end{array}$ & $\begin{array}{l}\text { Negocios } \\
\text { Comercio exterior } \\
\text { Publicidad } \\
\text { Servicios }\end{array}$ & $\begin{array}{l}\text { Actividades culturales } \\
\text { [Participación en] } \\
\text { Campeonatos } \\
\text { nacionales } \\
\text { Torneo Apertura } \\
\text { Copa Libertadores } \\
\text { Copa Intercontinental } \\
\text { Supercopa } \\
\text { Sudamericana } \\
\end{array}$ & $\begin{array}{l}\text { Actividad cultural } \\
\text { Capacitación } \\
\text { Servicios a los } \\
\text { afiliados } \\
\text { Servicios a la } \\
\text { comunidad }\end{array}$ & \begin{tabular}{|l|} 
Investigación \\
Extensión \\
Servicios a terceros
\end{tabular} & \begin{tabular}{|l|} 
Investigación \\
Licitaciones
\end{tabular} \\
\hline
\end{tabular}

TABLA 5

Espacio y tiempo

\begin{tabular}{|c|c|c|c|c|c|c|}
\hline Categoría & Vinos & Alimentos & Fútbol & Asociaciones & Facultades & Gobierno \\
\hline Espacio & $\begin{array}{l}\text { Argentina } \\
\text { Provincia de Mendoza } \\
\text { Ubicación geográfica } \\
\text { Origen }\end{array}$ & \begin{tabular}{|l|} 
Ambiente \\
Medio ambiente \\
Brasil \\
Chile \\
Perú \\
Latinoamérica \\
Internacional \\
\end{tabular} & - & - & Mapa de la Facultad & $\begin{array}{l}\text { Provincia de Buenos } \\
\text { Aires } \\
\text { Provincia de Santa Fe }\end{array}$ \\
\hline Tiempo & $\begin{array}{l}\text { Historia } \\
\text { Pasado y presente }\end{array}$ & Historia & $\begin{array}{l}\text { Historia } \\
\text { Personajes, perlas y } \\
\text { recuerdos } \\
\text { Números históricos }\end{array}$ & Historia & $\begin{array}{l}\text { Centenario de la } \\
\text { facultad }\end{array}$ & Historia del organismo \\
\hline
\end{tabular}

Sumando, de las 16 categorías del CRG (tabla 1), las bodegas de vino coincidieron en 13 (81\%), las industrias de alimentos en 11 (68\%), los clubes de fútbol en 7 (43\%), las asociaciones profesionales en 5 (31\%), las facultades en $8(50 \%)$ y las agencias de gobierno en 9 (56\%).

Como bien ha establecido el propio CRG, no todas las categorías son útiles o necesarias para todas las organizaciones, por ejemplo Entidades de la naturaleza o Entidades y organismos vivos difícilmente tengan relación con las asociaciones profesionales o las agencias de gobierno.

Por otro lado, es importante señalar también que los sitios analizados corresponden a los sitios Web públicos y no a la Intranet de las organizaciones, de manera que algunas categorías pueden no estar presentes, como por ejemplo Agentes (equipos o aparatos), o bien Partes o componentes. Otras categorías como Entidades abstractas, donde se han incluido rubros como filosofía corporativa, objetivos, visión, misión, liderazgo o políticas, pueden aplicarse a todo tipo de organización, aunque entre las que se analizaron sólo una las manifestó explícitamente.

\section{CONCLUSIONES}

Los sitios Web estudiados no han sido organizados deliberadamente por las categorías fundamentales del CRG, pero coinciden en muchas de ellas, según las características particulares de cada organización. Algunos grupos, si bien no hicieron explícitas algunas categorías, podían haberlas utilizado con beneficio.

En consecuencia, se puede concluir que las categorías fundamentales del CRG son adecuadas para analizar la información de las organizaciones y diseñar taxonomías corporativas, optimizando los sitios Web correspondientes. Resulta entonces indispensable para los bibliotecarios repasar los fundamentos del análisis por facetas $(9,21-22)$.

\section{Artigo recebido em 07-09-2004 e aceito para publicação de} 18 a $21 / 10 / 2004$.

\section{Agradecimientos}

Este trabajo ha sido subsidiado por el Programa de Incentivos del Ministerio de Educación de la Nación. Proyecto acreditado $n^{\circ} 11 \mathrm{H} / 361$. 
Las categorías o facetas fundamentales: una metodología para el diseño de taxonomías corporativas...

\section{REFERENCIAS}

1. TUDHOPE, D.; KOCH, T. (Ed.). Networked knowledge organization systems. Journal of Digital Information, v. 1, n. 8, 2001 Disponível em: 〈http://jodi.ecs.soton.ac.uk>. Acesso em: 4 abr. 2004

2. HILL, L.; KOCH, T. (Ed.). Networked knowledge organization systems. Journal of Digital Information, v. 4, n. 4, 2004. Disponível em: <http://jodi.ecs.soton.ac.uk>. Acesso em: 20 de jul. 2004.

3. HODGE, G. Systems of knowledge organization for digital libraries: beyond traditional authority files. Washington DC : Digital Library Federation, Council on Library and Information Resources, 2000. Disponível em: <http://www.clir.org/pubs/reports/pub91/contents.html. Acesso em: 2 oct. 2002.

4. KOCH, T.; DAY, M. The role of classification schemes in Internet resources description and discovery. London : UKOLN, 1999. Disponível em: <http://www.ukoln.ac.uk-metadata/desire/classification>. Acesso em: 3 sept. 2000.

5. ALMEIDA, M. B.; BOX, M. P. Una visão geral sobre ontologías: pesquisa sobre definições, tipos, aplicações, métodos de avaliação e de construção. Ciência da Informação, Brasília, v. 32, n. 3, p. 7-20, set./dez. 2003. Disponível em: <http://www.scielo.br/pdf/ci/v32n3/ 19019.pdf>. Acesso em: 10 jul. 2004.

6. GARCÍA JIMÉNEZ, A. Instrumentos de representación del conocimiento: tesauros versus ontologías. Anales de Documentación, v. 7, p. 79-95, 2004. Disponível em: <http://www.um.es/fccd/anales/ad07/ ad0706.pdf $>$. Acesso em: 18 mayo 2004.

7. REAL ACADEMIA ESPAÑOLA. Diccionario de la lengua española. 22. ed. Madrid, 2003. Disponível em: < http://www.rae.es>. Acesso em: 20 jul. 2004

8. GILCHRIST, A.; KIBBY, P. Taxonomies for business: access and connectivity in a wired world. London : TFPL, 2000.

9. RANGANATHAN, S. R. Prolegomena to library classification. 2. ed London : Library Association, 1957.

10.TUDHOPE, D. et al. Representation and retrieval in faceted systems Advances in Knowledge Organization, v. 8, p. 191-197, 2002. Disponível em: <http://www.glam.ac.uk/soc/research/hypermedia/publications/ presentationdocs/ISKOn.doc>. Acesso em: 4 abr. 2004

11. BROUGHTON, V. Facet analytical theory as a basis for a knowledge organization tool in a subject portal. Advances in Knowledge Organization, v. 8, p. 135-142, 2002. Disponível em: <http://www.ucl.ac.uk/factks/ paper2.htm>. Acesso em: 4 abr. 2004
12.LOUIS, A. J.; MADDOX, E. L.; WASHINGTON, W. Using faceted classification to provide structure for information architecture. In: ANNUAL INFORMATION ARCHITECTURE SUMMIT, 4., 2003, Portland OR. Meford NJ : [s. n.], 2003. Disponível em: <http:// depts.washington.edu/pettt/presentations/conf_2003/ IASummit.pdf>. Acesso em: 20 jul. 2004.

13.PRISS, U. Description logic and faceted knowledge representation. CEUR Workshop Proceedings, v. 22, 1999. Disponível em: <http:// www.upriss.org.uk/papers/d199.pdf>. Acesso em: 4 abr. 2004.

14.PRISS, U. Faceted information representación. In: STUMME, Gerd; AACHEN, Gerd. (Ed.). INTERNATIONAL CONFERENCE ON CONCEPTUAL STRUCTURES, 8., 2000. Proceedings... [S. 1.] : Shaker Verlag, 2000, p. 84-94. Disponível em: <http:// www.upriss.org.uk/papers/iccs00.pdf>. Acesso em 4 abr. 2004.

15.PRISS, U. A graphical interface for conceptually navigating faceted thesauri. Advances in Knowledge Organization, v. 6, p. 184-190, 1998. Disponível em: 〈http://www.upriss.org.uk/papers/isko98.pdf>. Acesso em: 4 abr. 2004.

16.PRISS, U.; JACOB, E. Utilizing faceted structures for information systems design. In: WOODS, Larry. (Ed.). ASIS ANNUAL MEETING, 62., 1999, Washington DC. Knowledge creation, organization, and use. Proceedings... Medford NJ, 1999, p. 203-212. <Disponível em: 〈http://www.upriss.org.uk/papers/asis99.pdf〉. Acesso em: 4 abr. 2004

17. BARITÉ ROQUETA, M. G. La noción de categoría y sus implicaciones en la construcción y evaluación de lenguajes documentales. In: CONGRESO ISKO- ESPANA EOCONSID, 4., 1999, Granada. La representación y la organización del conocimiento en sus distintas perspectivas: su influencia en la recuperación de la información. Actas... Granada : Facultad de Biblioteconomía y Documentación de la Universidad de Granada, 1999, p. 39-45.

18.FOSKETT, A. D. Subject approach to information. 5. ed. London : Bingley, 1995.

19.AITCHISON, J.; GILCHRIST, A.; BAWDEN, D. Thesaurus construction: a practical guide. 4. ed. London : Aslib, 2000, p. 68.

20.VICKERY, B. C. Faceted classification: a guide to construction and use of special schemes. London : Aslib, 1960.

21.KWASNIK, B. H. The role of classification in knowledge representation and discovery. Library Trends, v. 48, n. 1, p. 22-47, 1999.

22. SPITERI, L. A simplified model for facet analyses. Canadian Journal of Information and Library Science, v. 23, p. 1-30, 1998. Disponível em: <http://aifia.org/pg/a_simplified_model_for_facet_analysis.php>. Acesso em: 4 abr. 2004. 